\title{
Association of ciprofloxacin prescriptions to outpatients to Clostridium difficile infections
}

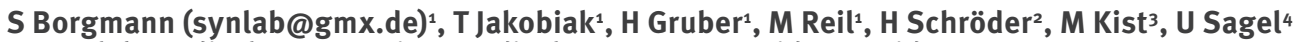

1. Synlab Medical Care Service, Medical Care Centre Weiden, Weiden, Germany

2. Wissenschaftliches Institut der Allgemeinen Ortskrankenkasse AOK (WidO, Research Institute of the AOK health

3. Department of Microbiology and Hygiene, University Hospital Freiburg, Freiburg, Germany

4. Austrian Agency for Health and Food Safety (AGES), Human Medicine, Vienna, Austria

Citation style for this article: Borgmann S, Jakobiak T, Gruber H, Reil M, Schröder H, Kist M, Sagel U. Association of ciprofloxacin prescriptions to outpatients to Clostridium difficile infections. Euro Surveill. 2010;15(5):pii=19479. Available online: http://www.eurosurveillance.org/ViewArticle.aspx?Articleld=19479

This article has been published on 4 February 2010

To study if antibiotic treatment of outpatients had triggered Clostridium difficile infections (CDI), prescription numbers were compared with CDI-affected patient numbers. A strong correlation was observed for ciprofloxacin $(R=0.917)$, suggesting that increased use of ciprofloxacin by outpatients contributed to increased numbers of CDI. These findings deserve further investigation as they may have an impact on future decisions regarding antibiotic prescribing.

Increasing numbers of Clostridium difficile infections (CDI) have been reported in North America and Europe over the past years. Use of antibiotics is widely acknowledged as a risk factor for hospitalised as well as outpatients [1]. In Germany increasing numbers of CDI are reported, while prescriptions of antibiotics for outpatients in general stayed constant. However, shifts from lower prescription rates of narrow-spectrum antibiotics to higher prescription rates of other antibiotics (e.g. quinolones, oral cephalosporins, combinations of aminopenicillins and beta-lactamase inhibitors) are obvious [2].

To study whether increased use of these antibiotics has contributed to increased numbers of CDI, antibiotic prescriptions to outpatients in southern Germany (Bavaria) were examined and compared to the number Tcd-positive patients identified in our laboratory. Here we describe that increased use of ciprofloxacin correlated with the number of Tcd positive patients suggesting that increased use of this drug might has contributed to increased numbers of CDI.

The number of antibiotic prescriptions was examined as described previously [3]. Prescription data from outpatients registered at statutory health insurances in Bavaria were obtained from the AOK Research Institute (WIdO) (http://www.wido.de). $83 \%$ of the Bavarian population are members of the statutory health insurances. Information on prescriptions for outpatients covered by all German statutory health insurances is collected by WIdO. Prescription data for the years 2000 to 2006 were calculated according to the World Health Organization's (WHO) Anatomical Therapeutic
Chemical (ATC) classification system (http://www. whocc.no). Prescriptions numbers are given in defined daily doses (DDD) per outpatient per three months.

The number of CDI-affected patients was assessed from the number of patients with $C$. difficile toxin (Tcd)positive stool samples tested in our microbiological department in Weiden in the period from 2000 to 2006, which serves about 40 hospitals and 2,500 ambulatory care settings (approximately 161,000 microbiological samples in 2006) [4].

The comparison of the number of Tcd-positive patients with the number of antibiotic prescriptions resulted in a negative correlation for class I cephalosporines (ATC index J01DB; Spearman's rank correlation coefficient $R=-0.446$ ), beta-lactamase-sensitive penicillins (J01CE; $R=-0.480$ ), combinations of sulfonamides and trimethoprim including derivatives (Jo1EE; $R=-0.563$ ), and macrolides (J01FA; -0.063). A positive correlation was noticed for second generation cephalosporins (J01DC; $R=0.711$ ), combinations of penicillins including beta-lactamase inhibitors (J01CR; $R=0.767$ ), and for quinolones $(\mathrm{J} 01 \mathrm{M} ; \mathrm{R}=0.709)$.

In contrast to second generation cephalosporins and combinations of penicillins with beta-lactamase inhibitors, certain quinolone derivatives are commonly used for the treatment of defined infections (e.g. moxifloxacin and levofloxacin for respiratory infections and ciprofloxacin for urinary infections). Therefore, a possible association of quinolone prescriptions with the number of Tcd-positive patients was analysed in more detail. From 2000 to 2006 , a continuous increase was noticed in the number of Tcd-positive patients, with an obvious peak in the first months of 2006 (Figure 1).

This course is paralleled by the prescription numbers of ciprofloxacin (Figure 2 ) resulting in a strong positive correlation coefficient of $\mathrm{R}=0.917$, p<0.0001 (Figure 3). Comparably low positive correlation coefficients were calculated for moxifloxacin $(R=0.382)$ and levofloxacin $(R=0.553$; data not shown). 
In contrast to moxifloxacin and levofloxacin, prescription numbers of ciprofloxacin exhibited only minor seasonal alterations suggesting that ciprofloxacin was not primarily used for the treatment of respiratory infections (Figure 2). The prescription rate of ciprofloxacin and folic acid antagonists taken together stayed fairly constant during the observation period [5]. So it appears obvious that ciprofloxacin is replacing trimethoprim-sulfamethoxazole (co-trimoxazole) and other folic acid antagonists for the treatment of urinary infections.

Recently it has been shown that consumption of cotrimoxazole by outpatients did not increase the risk of

\section{FIGURE 1}

Clostridium difficile toxin-positive patients (in- and outpatients), Bavaria, 2000-2006 (per three-month period) $(n=2,536)$

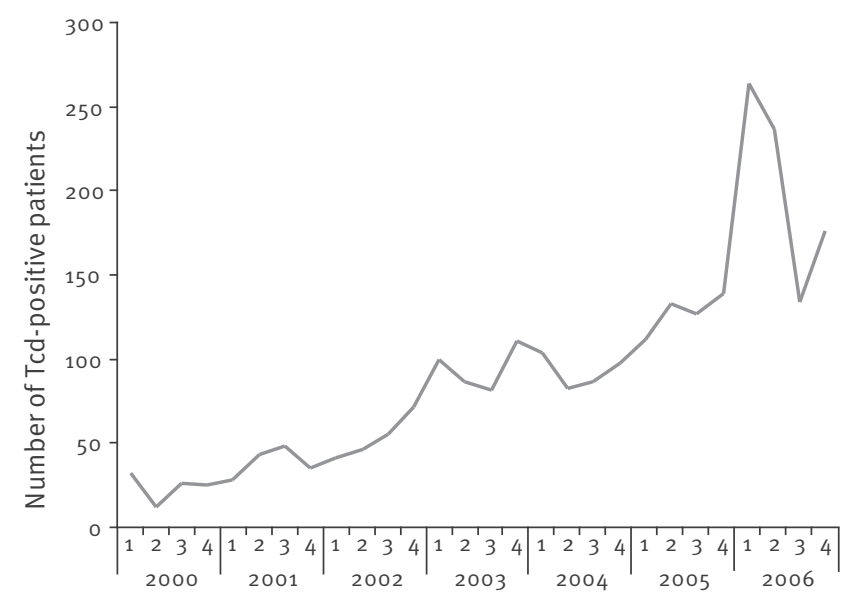

Date (in periods of three months)

Tcd: Clostridium difficile toxin.

\section{FIGURE 2}

Prescription of quinolone antibiotics to outpatients, Bavaria, 2000-2006 (per three-month period)

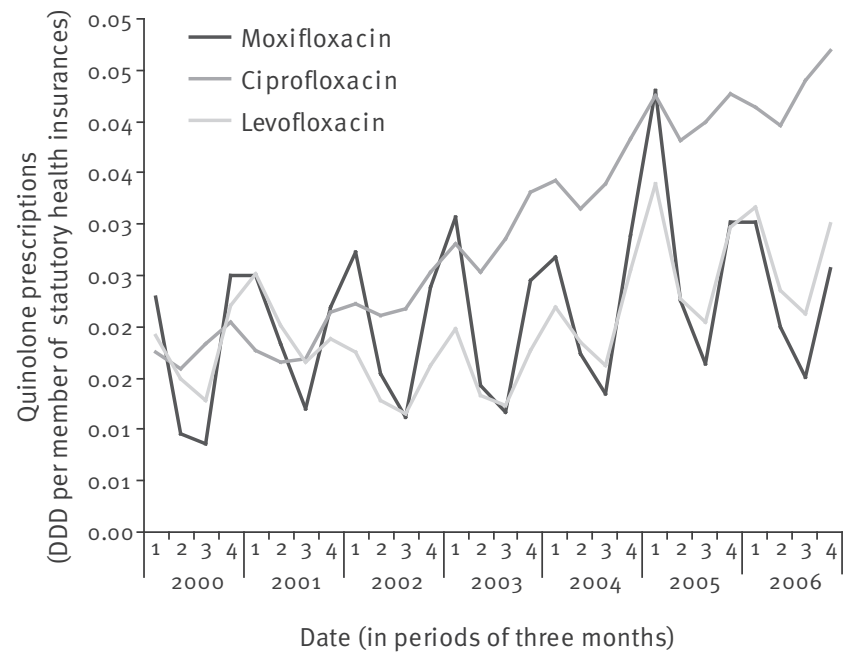

DDD: defined daily dose. developing CDI but the use of quinolones actually did $[1,6]$. Beside the well-known deleterious effects of CDI, application of quinolones seems to act as an up-regulating mediator for the production of toxins and germination of spores thus contributing to the epidemic spread of $C$. difficile $[7,8]$. Resistance to ciprofloxacin in C. difficile isolates from European patients is common and independent of bacterial ribotype, while resistance to moxifloxacine is restricted to defined ribotypes [4,9], suggesting that the increasing use of ciprofloxacin has indeed contributed to elevated numbers of CDI.

A limitation of our study might be that antibiotic prescriptions to outpatients were compared to the number of CDI-affected in- and outpatients. On the other hand, it is difficult to distinguish between community-acquired and hospital-acquired CDI. In a previous study, $44 \%$ of CDI-affected hospitalised patients developed symptoms and exhibited Tcd-positive stool samples within 48 hours after admission. Many of these patients had been hospitalised previously and were probably colonised with $C$. difficile at that time. When re-admitted later for infections other than CDI and treated with antibiotics, the treatment may then have induced the development of CDI [10]. Therefore, antibiotic treatment of outpatients, who acquired $C$. difficile at previous hospital stays could result in hospitalised and also in non-hospitalised patients suffering from CDI, which is a reason to include hospitalised CDI patients in the study. Moreover, even when restricting the analysis to CDI-affected outpatients, a positive correlation with ciprofloxacin prescription was observed $(R=0.744)$.

Nevertheless, also ciprofloxacin treatment of inpatients may be contributing to CDIs. Unfortunately, data about the use of antibiotics in German hospitals are not easily accessible, but there seems to be a parallel

\section{FIGURE 3}

Correlation of ciprofloxacin prescriptions to the number of Tcd-positive patients, Bavaria, 2000-2006 (per threemonth period)

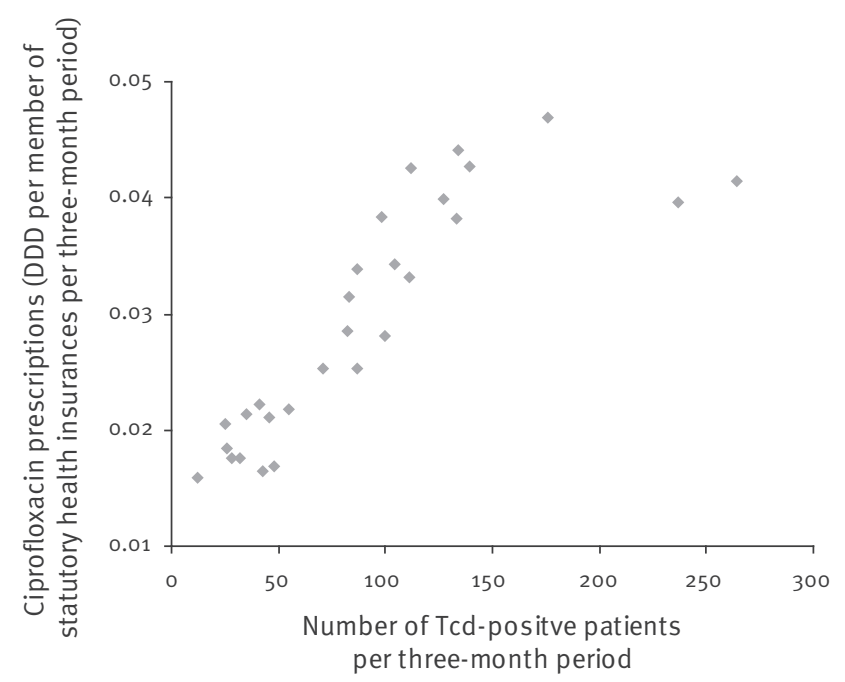

DDD: defined daily dose. 
development to that observed in ambulatory settings, with quinolones as the second most prescribed antibiotics in the hospital setting [11].

In summary, the present study shows that increasing consumption of ciprofloxacin by outpatients correlates with increasing numbers of CDI. Therefore, replacement of folic acid antagonists with ciprofloxacin for the treatment of urinary infections seems to be an underestimated mechanism for the propagation of $C$. difficile infections. This needs to be investigated further as it may have an impact on future decisions regarding antibiotic prescribing.

\section{References}

1. Dial S, Kezouh A, Dascal A, Barkun A, Suissa S. Patterns of antibiotic use and risk of hospital admission because of Clostridium difficile infection. CMAJ. 2008;179(8):767-72.

2. Kern WV, Schröder H. Antibiotic consumption in human medicine. 2.2. Antibiotic consumption in ambulatory settings. Bundesamt für Verbraucherschutz und Lebensmittelsicherheit, Paul-Ehrlich-Gesellschaft für Chemotherapie e.V., Infektiologie Freiburg, editors. Genars. Rheinbach (Germany): Antiinfectives Intelligence, Gesellschaft für klinisch-mikrobiologische Forschung und Kommunikation mbH; 2008. p. 11-16. German.

3. Borgmann S, Jakobiak T, Gruber H, Schröder H, Sagel U. Prescriptions of broad-spectrum antibiotics to outpatients do not match increased prevalence and antibiotic resistance of respiratory pathogens in Bavaria. Pol J Microbiol. 2009; 58(2):105-10.

4. Borgmann S, Kist M, Jakobiak T, Reil M, Scholz E, von EichelStreiber C, et al. Increased number of Clostridium difficile infections and prevalence of Clostridium difficile PCR ribotype 001 in southern Germany. Euro Surveill. 2008;13(49). pii=19057. Available from: http://www.eurosurveillance.org/ ViewArticle.aspx?Articleld=19057

5. Borgmann S, Jakobiak T, Gruber H, Schröder H, Sagel U. Ciprofloxacin treatment of urinary infections results in increased resistance of urinary E. coli to Ciprofloxacin and Cotrimoxazole. Pol J Microbiol. 2009;58(4):371-3.

6. Delaney JA, Dial S, Barkun A, Suissa S. Antimicrobial drugs and community-acquired Clostridium difficile associated disease, UK. Emerg Infect Dis. 2007;13(5):761-3.

7. Saxton K, Baines SD, Freeman J, O'Connor R, Wilcox MH. Effects of exposure of Clostridium difficile PCR ribotypes 027 and 001 to fluoroquinolones in a human gut model. Antimicrob Agents Chemother. 2009;53(2):412-20.

8. Adams DA, Riggs MM, Donskey CJ. Effect of fluoroquinolone treatment on growth of and toxin production by epidemic and nonepidemic clostridium difficile strains in the cecal contents of mice. Antimicrob Agents Chemother. 2007;51(8):2674-8.

9. Spigaglia P, Barbanti F, Mastrantonio P, Brazier JS, Barbut F Delmée $\mathrm{M}$, et al. Fluoroquinolone resistance in Clostridium difficile isolates from a prospective study of $C$. difficile infections in Europe. J Med Microbiol. 2008;57(Pt6):784-9.

10. Price MF, Dao-Tran T, Garey KW, Graham G, Gentry LO, Dhungana L et al. Epidemiology and incidence of Clostridium difficile-associated diarrhoea diagnosed upon admission to a university hospital. J Hosp Infect. 2007;65(1):42-6.

11. DeWith K, Kern WV. Antibiotic consumption in human medicine. 2.2. Antibiotic consumption in the hospital. In: Bundesamt für Verbraucherschutz und Lebensmittelsicherheit, PaulEhrlich-Gesellschaft für Chemotherapie e.V., Infektiologie Freiburg, editors. Genars. Rheinbach (Germany): Antiinfectives Intelligence, Gesellschaft für klinisch-mikrobiologische Forschung und Kommunikation mbH; 2008. p. 17-22. German. 